\title{
Relapsing Salmonella enteritidis infection in a young adult male with chronic granulomatous disease
}

\author{
A.F. Safe, R.T. Maxwell, A.J. Howard and R.C. Garcia ${ }^{1}$ \\ Department of Medicine and Microbiology, Gwynedd District General Hospital, Bangor, Gwynedd and \\ 'Department of Medicine, Faculty of Clinical Science, University College, London, WC1, UK
}

\begin{abstract}
Summary: A case of chronic Salmonella enteritidis infection in a young adult patient is reported. Following an initial enteritis with bacteraemia, the illness lasted for a 7-year period and was characterized by recurrent pyrexia, anaemia, weight loss and marked hepatosplenomegaly.

Investigations revealed that the patient suffers from a mild form of chronic granulomatous disease presenting in adolescence.
\end{abstract}

\section{Introduction}

Chronic granulomatous disease (CGD) is a rare syndrome characterized by recurrent, often prolonged purulent infections associated with impaired neutrophil bactericidal activity. The disease usually arises in infancy with a fatal outcome by adolescence. ${ }^{1}$ However, several cases of young adults with CGD have been reported ${ }^{2}$ and recently there has been increased recognition of the mild form of the disease. ${ }^{3}$

Infections are caused predominantly by catalasepositive micro-organisms such as staphylococci, Escherichia coli, Serrata spp., Aspergillus spp. and Candida albicans.

Described here is an unusual case of a young male patient with a mild form of CGD who presented with a chronic salmonella infection which ran a relapsing course over a 7 -year period.

\section{Case report}

A 20 year old male patient presented in 1980 with acute diarrhoea and high fever. Salmonella enteritidis phage type 8 was isolated from blood and faeces. He was treated with intravenous ampicillin for 10 days and parenteral fluid replacement. In 1984 he was readmitted with a few weeks' history of anorexia, general malaise, weight loss and recurrent epistaxis. On examination he was anaemic with a temperature of $38^{\circ} \mathrm{C}$. There were signs of consolidation at the lower lobe of the left lung with

Correspondence: A.F. Safe, M.Sc., M.R.C.P., Geriatric and General Medicine, Bristol Royal Infirmary, Bristol BS2 8HW, UK.

Accepted: 5 September 1990 pleural effusion. Laboratory investigations were as 옥 follows: haemoglobin $(\mathrm{Hb}): 8.6 \mathrm{~g} / \mathrm{dl}$ (normal range: 13.5-18), white cell count (WCC): $5.3 \times 10^{9} / 1 \frac{\mathbb{D}}{8}$ (normal range: $4-11$ ), platelet count: $700 \times 10^{9} / 1$ (normal range: 150-400) and erythrocyte sedimentation rate (ESR): $54 \mathrm{~mm} / \mathrm{h}$ (normal range: $0-58$ Monospot test was positive. Urea and electrolyte $\notin$ were normal and liver function tests showed mild raised alkaline phosphatase (ALP) $320 \mathrm{IU} / 1$ (no mal range: $30-120$ ). Blood and pleural fluid cultures were negative but pleural fluid examination revealed sheets of lymphocytes associated with atypical mononuclear cells suggestive of EpsteinBarr virus infection. He was transfused with 4 units of blood and his general condition improved. He was discharged and followed up in the outpatient clinic.

A year later he was readmitted for investigation of anaemia and recurrent pyrexia. Liver function tests showed a normal bilirubin level but elevated values of enzymes; serum aspartate transaminase: $54 \mathrm{IU} / 1$ (normal range: 8-40), ALP: 703 IU/l. Blood, stool and urine cultures were negative. Hepatitis A and B, brucellosis, toxoplasmosis, 우 cytomegalovirus infection and leptospirosis were all excluded by serological investigations. Trephine biopsy of the iliac crest showed an active marrow with non-caseating granulomata. He was transfused with 3 units of blood and followed up as an outpatient.

In March 1986 he was readmitted with fever, progressive weight loss and epistaxis. On examination he was pyrexial, there was marked anaemia and moderate hepatosplenomegaly. Liver biopsy? showed non-caseating granulomata. Anti-tuberculous therapy was commenced with ethambutol, isoniazid and rifampicin. His fever settled with 
clinical improvement and some regression of the hepatosplenomegaly but liver function tests continued to be abnormal with markedly elevated enzyme levels. Treatment was discontinued after 9 months in December 1986.

Three weeks later he presented again with fever, anaemia and weight loss. On this occasion $S$. enteritidis phage type 8 was isolated from both blood and stool. Co-trimoxazole $960 \mathrm{mg}$ twice daily was commenced with remarkable improvement clinically and biochemically. There was marked reduction of the hepatosplenomegaly. Endoscopic retrograde cholangiography showed normal gall bladder and biliary tree. Bile cultures were negative. Bone scan and human immunodeficiency virus (HIV) screening were negative. Repeated echocardiography showed no evidence of endocarditis. Haemoglobin electrophoresis and immunoglobulin studies were normal. There was a normal response to the streptokinase-streptodornase and candidin skin tests indicating intact cell mediated immunity. $T$ cell assay and complement studies were normal. The co-trimoxazole treatment was continued for 3 months. Three months after stopping treatment he relapsed and was admitted with fever and general malaise. $S$. enteritidis phage type 8 was again isolated from blood cultures. Co-trimoxazole was restarted and continued for another 6 months. He has now been clinically well for 18 months since stopping the antibiotics. On a recent physical examination the patient was well developed and the hepatosplenomegaly had fully resolved. His biochemical and haematological indices remain normal.

The patient's two siblings and parents were all in good health. The patient's maternal grandmother was in good health but his maternal grandfather had a life long history of skin infections and was said to have died of tuberculosis at the age of 62 .

The patient had neutrophil function studies as described below.

\section{Methods}

Preparation of polymorphonuclear neutrophils (PMNs) PMNs were isolated from the patient's blood by sedimentation of red cells in the presence of dextran, centrifugation of the supernatant on Ficoll-Sodium metrizoate (Lymphoprep, Nycomed UK) and hypotonic lysis of the red cells remaining in the granulocyte pellet. ${ }^{4}$

Cytochrome b-245 was detected on Western blots of sodium dodecylsulphate-polyacrylamide gels of a cell extract from the patient's neutrophils. Normal cell extracts were used for comparison. Extracts were prepared by sonication of hypotonic suspensions of neutrophils. The immune-reaction on the blots was carried out using a specific antibody against the $22 \mathrm{KDa}$ alpha subunit of the cytochrome. $^{5}$

Superoxide anion generation was measured as the rate of superoxide dismutase-inhabitable reduction of cytochrome $\mathrm{c}$, using phorbol myristate acetate $(200 \mathrm{ng} / \mathrm{ml})$ as the stimulus. ${ }^{6}$

Nitroblue tetrazolium (NBT) dye reduction Quantitative NBT determinations were performed by the method of Baehner and Nathan.

Aerobic and anaerobic leucocyte bactericidal activity This was performed as described by Mandell. ${ }^{8}$ Four $\mathrm{ml}$ of a PMN suspension containing $5 \times 10^{6}$ bacteria and $5 \times 10^{6} \mathrm{PMN}$ were used. Samples were removed at $0,30,60$ and 120 minutes. PMNs from healthy adults were obtained as controls and were always run parallel with the PMNs of the patients.

\section{Results}

Cytochrome $b$ The haem-containing alpha subunit of the cytochrome was not detectable immunochemically on Western blot.

Superoxide generation The patient's neutrophils generated superoxide at $1.3 \mathrm{nmol} / \mathrm{min} / 10^{7}$ cells, that is, approximately $1 \%$ of the normal rate.

Reduction of nitroblue tetrazolium When examined under the microscope, nearly all the phorbol myristate acetate-treated granulocytes from the patient reduced NBT. However, as shown in Table $\mathrm{I}$, the intensity of the reduction was low in comparison to the dark reaction products in control cells, and most of the cells presented only a faint blue granular appearance in their cytoplasm.

Staphylococcal bactericidal index Granulocytes from the patient showed defective in vitro bac-

Table I Reduction of nitroblue tetrazolium (NBT) by granulocytes of the patient and controls

\begin{tabular}{|c|c|c|c|}
\hline \multirow[b]{2}{*}{$\begin{array}{l}\text { Degree of NBT } \\
\text { reduction* }\end{array}$} & \multicolumn{3}{|c|}{ Subject (\% granulocytes) } \\
\hline & & $\begin{array}{l}\text { Normal } \\
\text { control }\end{array}$ & $\begin{array}{c}\text { Carrier } \\
\text { of } C G D \dagger\end{array}$ \\
\hline None & 1 & 1 & 45 \\
\hline Weak & 95 & 2 & 4 \\
\hline Strong & 4 & 97 & 51 \\
\hline
\end{tabular}

*Individual granulocytes were examined under direct microscopy. Lack of reduction was scored as 0 , weak reduction as 1 , and strong reduction as $2 ; \uparrow$ Mother of a patient with X-linked CGD. 
tericidal activity against Staphylococcus aureus $502 \mathrm{~A}$ on three occasions. In a typical experiment normal granulocytes killed nearly all bacteria by 60 minutes; $3 \%$ of colony-forming units still survived after 60 minutes, and $8 \%$ after 120 . The patient's granulocytes failed to kill $28 \%$ of bacterial colonyforming units after 60 minutes, and $75 \%$ after 120 minutes.

These results indicate that this patient suffers from a variant form of chronic granulomatous disease. ${ }^{9}$ It would appear that the ability of the phagocytes to generate a very low level of superoxide on stimulation is sufficient to result in a milder form of the disease.

\section{Discussion}

Systemic infection may follow salmonella gastroenteritis. This is more likely in immunosuppressed patients. In these cases chronic infection characterized by recurrent bacteraemia and clinical relapse may ensue. This has been particularly associated with Hodgkin's lymphoma, ${ }^{10}$ asplenism $^{11}$ and acquired immune deficiency syndrome. ${ }^{12}$ A further case is described here in an adolescent patient who had a mild form of CGD. He exhibited a chronic illness characterized by anaemia, weight loss and relapsing febrile episodes. Salmonella enteritidis phage type 8 was isolated from blood cultures on three separate occasions over a 7-year period. Partial resolution of symptoms and signs occurred following anti-tuberculous therapy, but there was a relapse after the drugs were stopped. More noticeable improvement was seen after a 3-month course of co-trimoxazole although relapse was again seen after cessation of the drug. A subsequent 6-month course was successful in eradicating the infection.

The diagnosis of tuberculosis was suggested by the presence of granulomata in the bone marrow

\section{References}

1. Johnston, R.B. \& Baehner, R.L. Chronic granulomatous disease. Correlation between pathogenesis and clinical findings. Pediatrics 1971, 48: 730 .

2. Dilworth, J.A. \& Mandell, G.L. Adults with chronic granulomatous disease of childhood. Am J Med 1977, 63: 233-243.

3. Tauber, A.L., Borregaard, N., Simons, E. \& Wright, J. Chronic granulomatous disease: a syndrome of phagocyte oxidase deficiencies. Medicine 1983, 62: 286-309.

4. Segal, A.W., Dorling, J. \& Coade, S. Kinetics of fusion of the cytoplasmic granules with phagocytic vacuoles in human polymorph-nuclear leucocytes. Biochemical and morphological studies. J Cell Biol 1980, 85: 42-59.

5. Segal, A.W. Absence of both cytochrome b subunits from neutrophils in $\mathrm{X}$-linked chronic granulomatous disease. Nature (London) 1987, 326: 88-91. and the liver. In retrospect these were also consistent with histological findings associated with $\mathrm{CGD}^{13}$ and chronic salmonellosis. ${ }^{14}$ It is likely that the response seen with anti-tuberculous therapy was due to rifampicin which is active against salmonella ${ }^{15}$ and has been used successfully in combination with other antibiotics for treating salmonella carriers. ${ }^{16}$

The aetiology of the chest infection and pleural effusion which occurred in 1984 is unclear. Pulmonary involvement in Epstein-Barr virus infection is rare. ${ }^{17}$ Salmonellosis has been noted to invoke the production of atypical mononuclear cells ${ }^{18}$ and it is more likely that the episode represented recurrence of salmonella infection with a false positive monospot test.

The reported patient suffers from a very rare variety of CGD. His cells did respond to maximal stimulation, but with a minute proportion of the normal production of superoxide. This would explain why he was unable to eradicate the salmonella infection but has been relatively well apart from that. Patients with a mild form of CGD usually present with skin infection, ${ }^{3}$ but our patient presented with recurrent invasive salmonellosis which, as far as we are aware, has not been previously described.

Recent study has demonstrated partial correc tion of the cellular defect in CGD by subcutaneous interferon gamma. ${ }^{19}$ The results of the study sug gest a potential role for interferon gamma in the prophylaxis of infections or as an adjunct to conventional antimicrobial therapy for acute infections in patients with CGD.

\section{Acknowledgements}

We would like to thank Professor A. Segal, Professor of Medicine at University College Hospital, London, for his assistance and Mrs M. Wright for preparing the manuscript.

6. Garcia, R.C., Cross, A.R. \& Segal, A.W. The development of cytochrome b-245 in maturing human macrophages. Biochem J 1986, 239: 647-651.

7. Baehner, R.L. \& Nathan, D.G. Quantitative nitroblue tetrazolium test in chronic granulomatous disease. $N$ Engl J Med 1968, 278: 971.

8. Mandell, G.L. Bactericidal activity of aerobic and anaerobic polymorphonuclear neutrophils. Infect Immunol 1974, 9: 337.

9. Ezekowitz, R.A.B., Orkin, S.H. \& Newburger, P.E. Recombinant interferon gamma augments phagocyte superoxide production and $\mathrm{X}$-chronic granulomatous disease gene expression in X-linked variant chronic granulomatous disease $J$ Clin Invest 1987, 80: 1009-1016.

10. Bolivar, R., Bodey, G.P. \& Valsues, W.S. Recurrent salmonella meningitis in a compromised host. Cancer 1982, 50 34-36. 
11. Zulma, A., Lewis, D. \& Brown, J. Ciprofloxacin treatment of recurrent Salmonella typhimurium septicaemia in a splenectomised and immunosuppressed patient. $J$ Antimicrob Chemother 1988, 21: 809-810.

12. Nadleham, R.B., Mather, W.U. \& Yancorits, S.R. Salmonella bacteria associated with the acquired immunodeficiency syndrome (AIDS). Arch Intern Med 1985, 145: $1968-1971$.

13. Bunch, C. Functional neutrophil disorders. In: Weatherall, D.J., Ledingham, J.G.G. \& Warrell, D.A. (eds) Oxford Textbook of Medicine. Oxford University Press, Oxford, 1987, pp. 159-160.

14. Christie, A.B. Food poisoning: salmonellosis. In: Christie, A.B. (ed) Infectious Diseases, Epidemiology and Clinical Practice. Churchill Livingstone, Edinburgh, 1987, pp. 2-37.

15. Burniat, W., Toppet, M. \& De Mol, P. Acute and recurrent salmonella infections in three children with chronic granulomatous disease. $J$ Infect 1980, 2: 263-268.
16. Bessudo, D., Heredia, A., Duarte, A. \& Bucio, R. Rifampicin in eradication of salmonella from the intestinal tract. Chemotherapy 1972, 17: 71.

17. Pullen, H. Infectious mononucleosis. Br Med J 1973, 1: 350-352.

18. Black, P.H., Lawrence, J.K. \& Swatz, M.N. Salmonellosis, a review of some unusual aspects. $N$ Engl J Med 1960, 262: 864-921.

19. Ezekowitz, R.A.B., Dinauer, M.C., Jaffe, H.S., Orkin, S.H. \& Newburger, P.E. Partial correction of the phagocyte defect in patients with $\mathrm{X}$-linked chronic granulomatous disease by subcutaneous interferon gamma. $N$ Engl J Med 1988, 319: 146-151. 